\title{
RADIO-FREQUENCY INTERFERENCE LOCATION, DETECTION AND CLASSIFICATION USING DEEP NEURAL NETWORKS
}

\author{
A. Pérez ${ }^{1}$, J. Querol ${ }^{2}$, H. Park ${ }^{1}$, and A. Camps ${ }^{1}$ \\ ${ }^{1}$ CommSensLab - UPC, Universitat Politècnica de Catalunya - BarcelonaTech, and IEEC/CTE-UPC \\ ${ }^{2}$ Interdisciplinary Centre for Security Reliability and Trust (SnT), University of Luxembourg
}

\begin{abstract}
Global Navigation Satellite System (GNSS) signals are used in Earth Observation for Radio Occultation and Reflectometry. The increasing effects of Radio-Frequency Interferences (RFI) on the performance of these receivers and navigation have suddenly sparked serious concerns due to their proliferation. Detection and mitigation of RFI heavily relies on the nature and location of the interfering sources. In some cases, null-steering or shielding can be used to mitigate RFI effects. In this work, a system to detect and locate RFI sources is presented, including signal classification and recording for countermeasure-related decision-making.
\end{abstract}

Index Terms- RFI, jamming, GNSS, GNSS-R, detection, mitigation.

\section{INTRODUCTION}

The use of Global Navigation Satellite System (GNSS) signals is becoming more ubiquitous due to the ever increasing services that rely on them for both positioning and timing, and more recently, for Earth Observation as well (e.g. GNSS-RO and GNSS-R).

Consumer uses such as navigation, absolute timing, geofencing or photographic geocoding account for the largest number of uses of GNSS signals. The interruption of such GNSS services due to Radio-Frequency Interference (RFI) is a nuisance with an invaluable economic impact [1].

Detection, location and recording of RFI signals is needed for long-term characterization of interference events and to develop mitigation strategies. A proof-of-concept version of a system capable of the aforementioned technique can be found in $[2,3]$, where a GNSS L1/E1 band with $2 \mathrm{MHz}$ bandwidth detection and location system was built. In this work, the design and development of an improved version with 100 $\mathrm{MHz}$ bandwidth is presented, with emphasis in detection algorithms for unknown RFI signals in GNSS bands, geolocation of RFI sources, and classification of interference events using Machine Learning techniques.

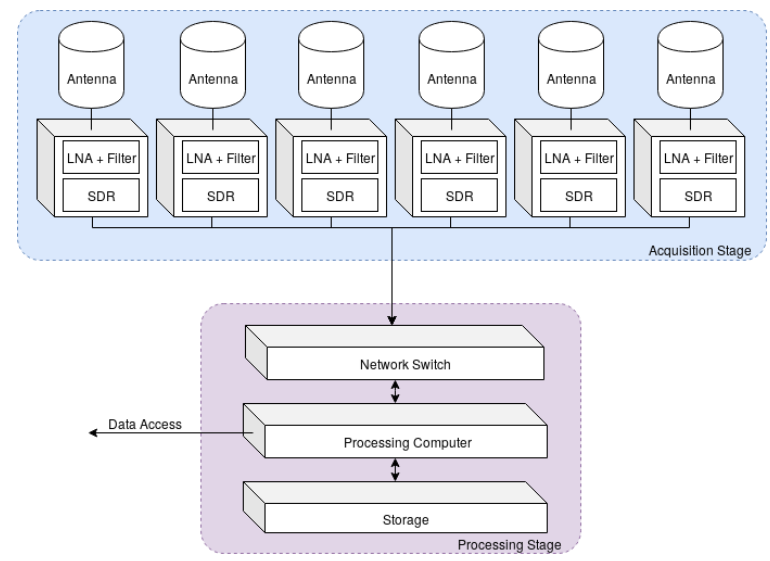

Fig. 1: System architecture for the proposed RFI Detector

\section{RFI DETECTOR AND LOCATOR}

In order to provide valuable data for the development of mitigation strategies in GNSS receivers, a representative system has to be used. GNSS signals for civilian use are found in $1,575.42 \mathrm{MHz}$, so a transceiver capable of working at that frequency should be at the core. In order to take into account outof band emissions that might hinder the performance of such receivers, a bandwidth wider than the typical $24 \mathrm{MHz}$ used for GPS is used. Software-Defined Radios (SDR) that work at the aforementioned frequencies with a bandwidth larger than 24 $\mathrm{MHz}$ are scarce, but Avnet provides several low-cost options capable enough, such as the PicoZED SDR [4]. For site surveys, Angle of Arrival measurements are extremely important, both for detection of RFI sources and for null-steering techniques. Thus, a uniform circular array (UCA) of antennas will be used for coarse AoA determination. The architecture of the proposed system is comprised of two high-level blocks, as seen in Fig. 1: the acquisition sub-system (AS) and the processing sub-system (PS).

In order to provide coarse location of the RFI source, 6 sectorial antennas are used in the AS. This static setup allows for a low-budget determination of the coarse Angle of Arrival (AoA) by comparing the power of the RFI in adjacent sectors. Each antenna has a $60^{\circ}$ beamwidth at $-3 \mathrm{~dB}$ in the horizontal and vertical planes. These sectorial elements allow for the 


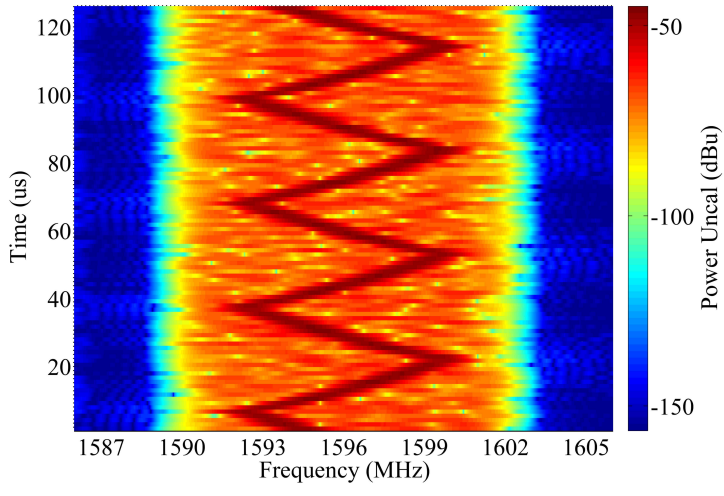

Fig. 2: Third Harmonic of DDR2 RAM memory causing unintentional Chirp-like RFI at $1.596 \mathrm{MHz}$

determination of the AoA with $60^{\circ}$ of resolution in azimuth, or better if the AoA is estimated for the detected RFI (i.e. with the same time-frequency properties) from the ratio of the powers detected in adjacent sectors in a technique known as Power Difference of Arrival (PDOA). The wide beamwidth in elevation ensures that all RFI in the horizontal plane are captured regardless of the distance from a terrestrial RFI source to the system or even if the RFI source is airborne. For this implementation, helical antennas providing right-handed circular polarization (RHCP) are used.

The signal from each antenna is then fed to an RF frontend that filters and amplifies the signals. Signal conditioning is performed by a Low-Noise Amplifier (LNA) located as close as possible to the antenna to minimize the overall Noise Figure (NF) of the system, followed by a band-pass filter to reject undesired out-of-band signals. The SDRs used are based on a commercial Analog Devices transceiver, coupled with a Xilinx Zynq FPGA for signal processing. The receiving frequency can be tuned within the range of GPS L1/ Galileo E1 $\pm 50 \mathrm{MHz}$, and it supports a sustained processing bandwidth of up to $5 \mathrm{MSps}$. The SDR will sample at $2 \mathrm{MSps}$ for $1 \mathrm{~ms}$ with a scanning strategy, looping through the 100 $\mathrm{MHz}$ band of interest, the output of which will then be sent to the PS for RFI detection and location.

In the PS, a computer processes the samples with RFI detection and location algorithms, classifies the RFI events, and provides a comprehensive report on the current situation.

\subsection{Detection}

RFI detection determines if an interference signal (Fig. 2) is present at any of the antennas of the system. For this purpose, two simple RFI detection algorithms are proposed: Kurtosis [5, 6] (Normality Test) and Spectrogram-based detection [7]. Kurtosis is a statistical fourth-order derived moment able to determine if the received signal (GNSS plus thermal noise) contains any non-Gaussian signal. Spectrogram-based detection is used to sense if there are spectrum anomalies. Both
RFI detection algorithms are used to determine if an RFI signal is present at any of the 6 antennas. These algorithms are well known and used in the literature [8]. In non-RFI scenario, signals from antenna must be Gaussian and with a calibrated and expected spectrum, which will depend on the center frequency. A threshold value, set according to a constant false alarm rate, will decide if there is RFI or not.

\subsection{Location}

RFI sectorial location determines the AoA of a detected interference signal. When an RFI signal is detected, the sectorial RFI location takes place. The $60^{\circ}$ sector which presents the larger power, among the ones where an RFI signal is detected, is determined to be the AoA. The current approach allows location of a single RFI per sector, which may be due to a single RFI source or the aggregate of several which fall into the same $60^{\circ}$ sector. In any case, the determined AoA will be the sector that corresponds to the maximum received RFI power. A better estimation can be achieved by calculating the ratio of the powers detected for the same RFI event in multiple sectors (Fig. 3). Using PDOA, the resolution of the AoA depends directly on the precision of the calculation of the radiation pattern for the antennas, as well as the number of bits of the Analog to Digital Converter used. A better but more complex alternative would be to synchronize the different SDR receivers, allowing for coherent reception of the signals and more precise AoA estimation using phase differences.

\subsection{Classification}

Parameters such as time duration, bandwidth, and duty cycle are estimated in this step to identify and classify the RFI signals. Usually, RFI signals have their power concentrated in either time, frequency, or both domains, in order to maximize the instantaneous and/or spectral power density [9]. Related to this classification, several pre-correlation techniques exist in different domains that are better detectors against certain types of RFI:

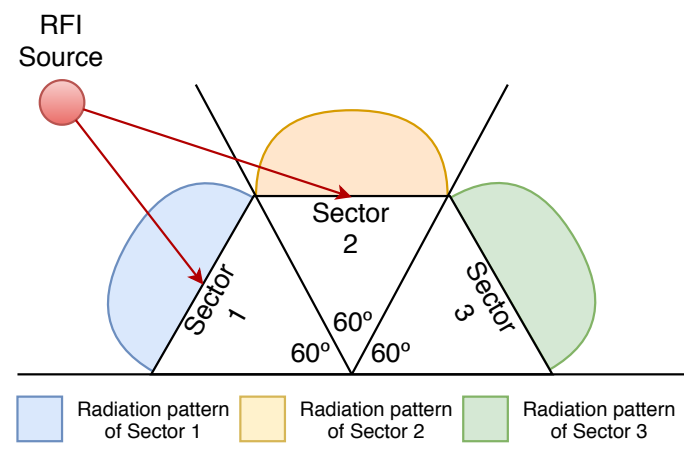

Fig. 3: Power Difference of Arrival method in a 3-sector configuration 


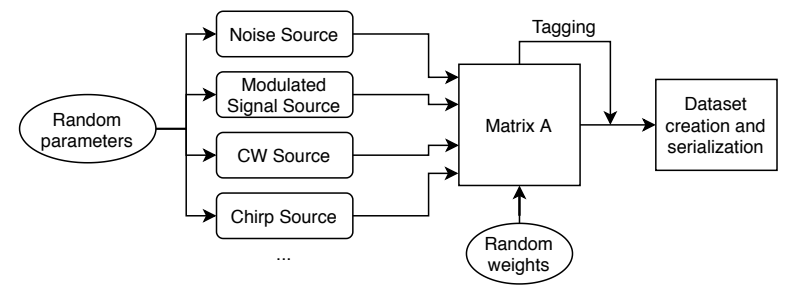

Fig. 4: Training and testing dataset generation

- Time domain techniques: One of the simplest techniques that consists in sampling the received signal and comparing its power with a threshold $\alpha$ directly related to the power of the signal in the case where no RFI exists. This kind of excision is adequate for strong and short bursts of RFI, whereas weak and long-lasting RFI may pass undetected. Some examples of this techniques are Pulse Blanking and Amplitude Domain Processing.

- Frequency domain techniques: The non-parametric methods consist on comparing the spectrum of the received signal with a theoretical threshold $\alpha$. These techniques have the same effect as a notch filter. This approach is not useful for non-stationary interference removal, as signals can change quickly in time. Notch Filtering and Frequency Blanking are the most used.

- Time-Frequency space techniques: They are the most used for detection and mitigation of RFI. These techniques take into account the fact that RFI spans both the time and frequency domain, thus being able to detect Continuous Wave and pulsed signals simultaneously. Since GPS signals are low power and wideband signals, the high power jammer signals can usually be distinguished very easily in time-frequency space. Some examples include Spectrogram Blanking and Filter Banks.

Even though Time-Frequency space techniques, and specially the Multiresolution Fourier Transform (MFT) [3] can be used as a generic mitigation technique with good results for each type of RFI, a truly optimal solution would be to classify each RFI event on-the-fly, and apply the best suited thresholding technique. The output of this classification will then be used to generate reports on RFI events, taking into account if the characteristics of two events separated in time are similar enough to classify them as the same RFI source, for example. This will allow for analysis of patterns in time, deployment of countermeasures against pervasive types of RFI, and improvements of the accuracy of the classification system.

\subsection{Testing}

In order to properly test the RFI detection and classification algorithms, synthetic RFI data has to be generated and fed into the system for verification. As seen in Fig. 4, a software consisting on several types of signal source such as noise, $\mathrm{CW}$, and various modulations that were linearly combined in a randomized fashion (with varying powers, frequencies, and parameters), was developed to properly test detection and classification processes. As the contents of each testing set were tagged for algorithm refinement and verification purposes, this development led to further research in the field of Machine Learning.

\section{DEEP LEARNING FOR RFI CLASSIFICATION}

Thanks to the recent advances in customized hardware (GPUs), as well as the rapid development of open-source tools, one approach to classification that has surged recently is the use of machine learning. Previous works have explored RFI detection for post-processing radio astronomy data [10], where astronomical signals are broad-band and vary smoothly over long time-scales.

In this work, classification of RFI signals for posterior excision in GNSS systems is explored by using the RFI generator developed in Sec. 2.4 to create a dataset. The basis for a performing machine learning system is to create a big, properly tagged sample database, which can be used to infer patterns from the data, from which classification can be attempted. Deep learning inference once the network has been trained allows for classification to occur with linear transformations on the input data, by applying a set of weights. This would allow for the fast RFI classification needed to choose the optimal mitigation strategy, as discussed in Section 2.3.

When training the neural network, it is imperative to understand the nuances of the classification that is to be attempted: that is, which features from the input data are more relevant to decide from the different classes into which the data is divided. In the case of RFI signals in GNSS environments, where normality tests (i.e. Kurtosis) are usually performed to discern RFI from Gaussian-like GNSS signals, their value becomes a great source of information to easily skip non-contaminated samples from decision. Due to the inherent characteristics of most jamming signals targeting the different domains discussed in Section 2.3, they can be spotted in a spectrogram or Fourier transform by having powers well over the noise floor. Thus, the Fourier transform will be the main source of data for the network, by containing valuable information to recognize RFI signals.

With this, a neural network (Fig. 5) was created to ingest

Table 1: Convolutional Neural Network structure

\begin{tabular}{lccc}
\hline Layer & Input size & Parameters & Activation function \\
\hline Convolutional 1 & $2 \times 4096$ & 16 filters & ReLU \\
\hline Convolutional 2 & 16x2x4096 & 32 filters & ReLU \\
\hline Fully connected 1 & 32768 & 4096 neurons & Softmax \\
\hline Fully connected 2 & 4096 & 512 neurons & Softmax \\
\hline Fully connected 3 & 512 & 3 neurons & Softmax \\
\hline
\end{tabular}




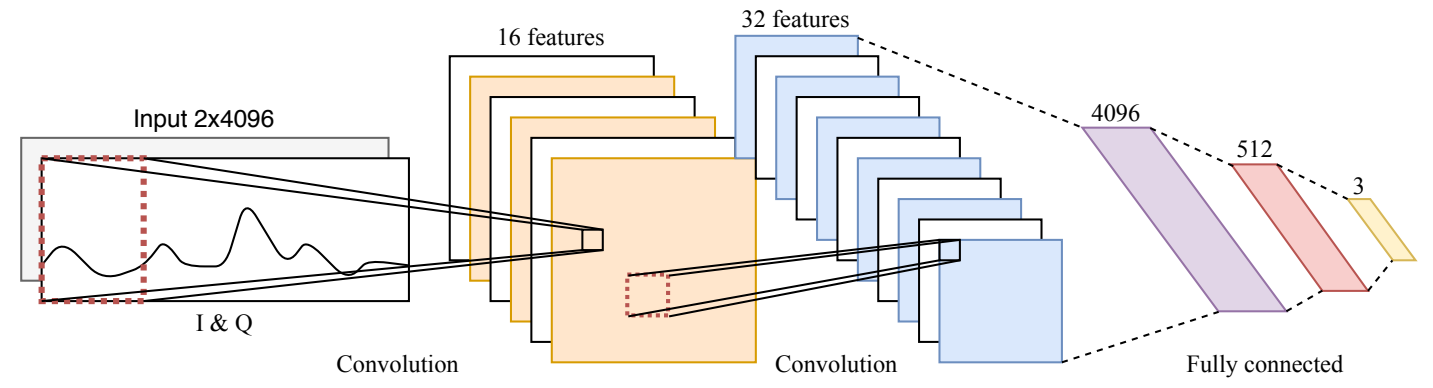

Fig. 5: RFI Classification Neural Network

samples tagged with the types of RFI contained within, which in this first version of the network are only of one of three signal types: noise, noise plus a continuous wave and noise plus a modulated signal.

A very simple neural network was created to test basic classification. The I\&Q samples are first convolved twice to add depth to the network, with kernel sizes that allow for the identification of features spanning as little as 3 bins, as seen in Table 1. After each convolution, a rectifier ReLU activation function is used. Finally, 3 fully connected layers are used to reduce the number of outputs in steps until the final 3 classes are reached. Training this network with only the 3 RFI types mentioned above caused significant overfitting to occur, resulting in poor performance, remarkedly worse than the algorithms used for classification.

\section{CONCLUSION}

Deep learning for RFI shows incredible potential both due to the availability of training examples that can be generated ondemand, and due to the different nature of RFI signals that can be classified with the correct information. Training and tuning of the neural network is still ongoing, with further information sources and a more diverse dataset to solve the overfitting problems that were found.

\section{ACKNOWLEDGEMENTS}

This work has received funding from project Spanish Ministerio de Ciencia, Innovación y Universidades and EU ERDF project ref. RTI2018-099008-B-C21 "SENSING WITH PIONEERING OPPORTUNISTIC TECHNIQUES“, and from the Korea Institute of Science and Technology (KIST) project "Monitoring and Mitigation of RFI Effects for GNSS Performance Improvement", and support grant MDM-2016-0600 to "María de Maeztu Excellence Units" CommSensLab, from Spanish Ministerio de Economía y Competitividad (MINECO/FEDER). This work was also supported by the Fond National de la Recherche Luxembourg, under the CORE project COHESAT: Cognitive Cohesive Networks of Distributed Units for Active and Passive Space Applications.

\section{REFERENCES}

[1] A. O'Connor and M. Gallaher, "Economic benefits of the Global Positioning System (GPS)," 062019.

[2] G. Forte, Contributions to radio frequency interference detection and mitigation in Earth observation, Ph.D. thesis, UPC, Jun 2013, Available at: https://www.tdx.cat/handle/10803/285320.

[3] J. Querol, Radio Frequency Interference Detection and Mitigation Techniques for Navigation and Earth Observation, Ph.D. thesis, UPC, 2018, Available at: https://www.tdx.cat/handle/10803/663905.

[4] PicoZED, "http://zedboard.org/product/picozed," .

[5] J. M. Tarongí, Radio Frequency Interference in Microwave Radiometry: Statistical Analysis and Study of Techniques for Detection and Mitigation, Ph.D. thesis, UPC, Mar 2012, Available at: https://www.tdx.cat/handle/10803/117023.

[6] R. D. De Roo, S. Misra, and C. S. Ruf, "Sensitivity of the Kurtosis Statistic as a Detector of Pulsed Sinusoidal RFI," IEEE Transactions on Geoscience and Remote Sensing, vol. 45, no. 7, pp. 1938-1946, jul 2007.

[7] J. M. Tarongi and A. Camps, "Radio frequency interference detection and mitigation algorithms based on spectrogram analysis," Algorithms, vol. 4, no. 4, pp. 239$261,2011$.

[8] J. M. Tarongi and A. Camps, "Normality Analysis for RFI Detection in Microwave Radiometry," Remote Sensing, vol. 2, no. 1, pp. 191-210, dec 2009.

[9] J. Querol, A. Perez, and A. Camps, "A Review of RFI Mitigation Techniques in Microwave Radiometry," Remote Sensing, vol. 11, no. 24, 2019.

[10] X. Zhang and T. Seyfi, "Deep Learning for Interference Identification: Band, Training SNR, and Sample Selection," 2019, Available at: https://arxiv.org/abs/1905.08054v1. 